Pacific Journal of Mathematics

TRANSFORMATIONS OF CERTAIN SEQUENCES OF RANDOM
VARIABLES BY GENERALIZED HAUSDORFF MATRICES 


\section{TRANSFORMATIONS OF CERTAIN SEQUENCES OF RANDOM VARIABLES BY GENERALIZED HAUSDORFF MATRICES}

\section{DAVID BORWEIN AND AMNON JAKIMOVSKI}

\section{Sufficient conditions are established for a generalized Hausdorff} matrix to transform certain sequences of random variables into almost surely convergent sequences.

1. Introduction. Suppose that $\left\{X_{n}\right\}(n=0,1, \ldots)$ is a sequence of random variables defined on a probability space $(\Omega, \mathcal{F}, P)$, and that $A=\left\{a_{n k}\right\}(n, k=0,1, \ldots)$ is an infinite matrix. Let

$$
T_{n}=\sum_{k=0}^{\infty} a_{n k} X_{k} .
$$

The following theorem concerning the almost sure convergence to zero of the sequence $\left\{T_{n}\right\}$ is due to Borwein [1].

Theorem A. If $1<p \leq 2,0<M<\infty$ and

(1) $\left|X_{n}\right| \leq M$ a.s. for $n=0,1, \ldots$,

(2) $\Sigma_{0 \leq i_{1}<i_{2}<\cdots<i_{n}}\left|E\left(X_{i_{1}} X_{i_{2}} \cdots X_{i_{n}}\right)\right|^{p /(p-1)} \leq M^{n}$ for $n=1,2, \ldots$,

(3) $\sum_{k=0}^{\infty}\left|a_{n k}\right|<\infty$ for $n=0,1, \ldots$, and

$$
\lim _{n \rightarrow \infty} \log n\left(\sum_{k=0}^{\infty}\left|a_{n k}\right|^{p}\right)^{1 /(p-n)}=0
$$

then $T_{n} \rightarrow 0$ a.s.

The sequence $\left\{X_{n}\right\}$ is said to be multiplicative if the expectation $E\left(X_{i_{1}} X_{i_{2}} \cdots X_{i_{n}}\right)=0$ whenever $0 \leq i_{1}<i_{2}<\cdots<i_{n}$; in particular, it is multiplicative if it is independent with $E X_{n}=0$ for $n=0,1, \ldots$ Condition (2) is trivially satisfied when $\left\{X_{n}\right\}$ is multiplicative. The nature of Theorem A is clarified by comparison with Kolmogorov's classical strong law of large numbers which states that if $\left\{X_{n}\right\}$ is independent with $E X_{n}=0$ for $n=0,1, \ldots$, and if

$$
\sum_{k=0}^{\infty} \frac{E X_{k}^{2}}{(k+1)^{2}}<\infty, \text { then } \frac{1}{n+1} \sum_{k=0}^{n} X_{k} \rightarrow 0 \text { a.s. }
$$

We shall denote by $\Gamma_{p}$ the set of matrices $A$ such that $T_{n} \rightarrow 0$ a.s. whenever the sequence $\left\{X_{n}\right\}$ satisfies conditions (1) and (2). Our primary 
object in this paper is to establish conditions which are both sufficient and easy to verify for generalization Hausdorff matrices to be in $\Gamma_{p}$. Included in the class of generalized Hausdorff matrices are the matrices of such well-known methods of summability as the Cesàro, the Euler, and the weighted mean methods.

The matrix $A$ is said to have the Borel property and we write $A \in(B P)$, if almost all sequences of zeros and ones are $A$-convergent to $1 / 2$. This amounts to (see [5])

$$
\frac{1}{2} \sum_{k=0}^{\infty} a_{n k}\left(1-X_{k}\right) \rightarrow \frac{1}{2} \quad \text { a.s. }
$$

when $\left\{X_{n}\right\}$ is the sequence of Rademacher functions on $\Omega=[0,1]$ and $P$ is Lebesgue measure. Since, in this case, $\left\{X_{n}\right\}$ satisfies conditions (1) and (2), it follows that

if $\sum_{k=0}^{\infty} a_{n k}$ is convergent for $n=0,1, \ldots$ and $\lim _{n \rightarrow \infty} \sum_{k=0}^{\infty} a_{n k}=1$, and if $A \in \Gamma_{p}$, then $A \in(B P)$.

Generalized Hausdorff matrices. Suppose in all that follows that $\lambda=\left\{\lambda_{n}\right\}$ is a sequence of real numbers satisfying

$$
\lambda_{0} \geq 0, \quad \lambda_{n}>0 \quad \text { for } n=1,2, \ldots, \lambda_{n} \rightarrow \infty, \quad \sum_{r=1}^{\infty} \frac{1}{\lambda_{n}}=\infty,
$$

and that $\alpha$ is a function of bounded variation on $[0,1]$.

For $0 \leq k \leq n, 0<t \leq 1$, let

$$
\begin{aligned}
& \lambda_{n k}(t)=-\lambda_{k+1} \cdots \lambda_{n} \frac{1}{2 \pi i} \int_{C} \frac{t^{2} d z}{\left(\lambda_{k}-z\right) \cdots\left(\lambda_{n}-z\right)} ; \\
& \lambda_{n k}(0)=\lambda_{n k}(0+),
\end{aligned}
$$

$C$ being a positively sensed closed Jordan contour enclosing $\lambda_{k}$, $\lambda_{k+1}, \ldots, \lambda_{n}$. We observe the convention that products such as $\lambda_{k+1} \cdots \lambda_{n}$ $=1$ when $k=n$. Let

$$
\lambda_{n k}=\int_{0}^{1} \lambda_{n k}(t) d \alpha(t) \quad \text { for } 0 \leq k \leq n ; \quad \lambda_{n k}=0 \text { for } k>n,
$$

and denote the triangular matrix $\left\{\lambda_{n k}\right\}$ by $H(\lambda, \alpha)$. This is called a generalized Hausdorff matrix.

Let

$$
\begin{aligned}
& D_{0}=\left(1+\lambda_{0}\right) d_{0}=1 \\
& D_{n}=\left(1+\frac{1}{\lambda_{1}}\right) \cdots\left(1+\frac{1}{\lambda_{n}}\right)=\left(1+\lambda_{n}\right) d_{n} \text { for } n \geq 1 .
\end{aligned}
$$


Then, for $n \geq 0$,

$$
D_{n}=\lambda_{n+1} d_{n+1}=\frac{\lambda_{0}}{1+\lambda_{0}}+\sum_{k=0}^{n} d_{k}
$$

It is known (see [3]) that

$$
\begin{gathered}
0 \leq \lambda_{n j}(t) \leq \sum_{k=0}^{n} \lambda_{n k}(t) \leq 1 \quad \text { for } 0 \leq t \leq 1,0 \leq j \leq n, \\
\int_{0}^{1} \lambda_{n k}(t) d t=\frac{d_{k}}{D_{n}} \quad \text { for } 0 \leq k \leq n, \\
\sum_{k=0}^{n}\left|\lambda_{n k}\right| \leq \int_{0}^{1}|d \alpha(t)| .
\end{gathered}
$$

Let

$$
\rho_{n k}=\sum_{j=k}^{n} \frac{1}{\lambda_{j}}, \quad \sigma_{n k}=\left(\sum_{j=k}^{n} \frac{1}{\lambda_{j}^{2}}\right)^{1 / 2} \quad \text { for } 1 \leq k \leq n .
$$

We shall prove the following theorems.

THEOREM 1. Let $M, m$ be positive constants. If $\alpha(0+)=\alpha(0)$ and $\alpha(1-)=\alpha(1)$, and if $\lambda$ satisfies either

$$
M \log \lambda_{k} \geq \lambda_{k+1}-\lambda_{k} \geq m \text { for.all sufficiently large } k
$$

or

(11) $M \geq \lambda_{k+1}-\lambda_{k}>0$ for all sufficiently large $k$ and $\log n / \sqrt{\lambda_{n}}=o(1)$, then $H(\lambda, \alpha) \in \Gamma_{2}$. If, in addition, $\alpha(1)-\alpha(0)=1$, then $H(\lambda, \alpha) \in(B P)$. either

TheOREM 2. Let $\alpha(t)=\int_{0}^{t} \beta(u) d u$ for $0 \leq t<1$, and let $1<p \leq 2$. If

$$
\beta \in L^{p}[0,1] \text { and } \max _{0 \leq k \leq n} d_{k} \cdot \frac{\log n}{D_{n}}=o(1)
$$

or

$$
\beta \in L^{\infty}[0,1] \text { and } \log n\left(\sum_{k=0}^{n}\left(\frac{d_{k}}{D_{n}}\right)^{p}\right)^{1 /(p-1)}=o(1)
$$

then $H(\lambda, \alpha) \in \Gamma_{p}$. If, in addition, $\left\{\lambda_{n}\right\}$ is non-decreasing and $\alpha(1)=1$, then $H(\lambda, \alpha) \in(B P)$. 
It is known that $H(\lambda, \alpha) \in(B P)$ when $\alpha$ satisfies the conditions of Theorem 1 and $\lambda_{n}=n+c$, the case $c=0$ of this result being due to Hill [6] and the case $c>0$ to Liu and Rhoades [9]. On the other hand, Borwein and Cass [2] have shown that $H(\lambda, \alpha) \notin(B P)$ when $\alpha(t)=t$ and $\lambda_{n}=$ $c \log (n+1), 0<c<1 / \log 4$. Borwein and Cass [2] have also shown Theorem 2 to hold in the case $p=2,0=\lambda_{0}<\lambda_{1}<\lambda_{2}<\cdots$.

\section{Preliminary results.}

LEMMA 1. If $1 \leq k \leq n, 0<\lambda_{k} \leq \lambda_{k+1} \leq \cdots \leq \lambda_{n}$ and $0 \leq t \leq 1$, then

$$
\lambda_{n k}(t) \leq \frac{\sqrt{2}}{\lambda_{k} \sigma_{n k}} .
$$

Proof. Since $0 \leq \lambda_{n k}(t) \leq 1$, we may suppose that

$$
\lambda_{k}^{2} \sum_{j=k}^{n} \frac{1}{\lambda_{j}^{2}}>2 .
$$

Jakimovski [6, Lemma 2.1] has shown that, for $u>0$,

$$
\lambda_{n k}\left(e^{-u}\right)=\frac{1}{2 \pi \lambda_{k}} \int_{-\infty}^{\infty} \frac{e^{i u v} d v}{\prod_{j=k}^{n}\left(1+i v / \lambda_{J}\right)},
$$

from which it follows that

$$
\lambda_{n k}\left(e^{-u}\right) \leq \frac{1}{2 \pi \lambda_{k}} \int_{-\infty}^{\infty} \frac{d v}{\prod_{j=k}^{n}\left(1+v^{2} / \lambda_{j}^{2}\right)^{1 / 2}} .
$$

Next, we have, by (14), that

$$
\begin{aligned}
\prod_{j=k}^{n}\left(1+\frac{v^{2}}{\lambda_{j}^{2}}\right) & \geq 1+v^{2} \sum_{j=k}^{n} \frac{1}{\lambda_{j}^{2}}+\frac{v^{4}}{2} \sum_{r=k}^{n} \frac{1}{\lambda_{r}^{2}}\left(\sum_{j=k}^{n} \frac{1}{\lambda_{j}^{2}}-\frac{1}{\lambda_{r}^{2}}\right) \\
& \geq 1+v^{2} \sum_{j=k}^{n} \frac{1}{\lambda_{j}^{2}}+\frac{v^{4}}{4} \sum_{r=k}^{n} \frac{1}{\lambda_{r}^{2}} \sum_{j=k}^{n} \frac{1}{\lambda_{j}^{2}}=\left(1+\frac{v^{2}}{2} \sigma_{n k}^{2}\right)^{2}
\end{aligned}
$$

Hence, for $u>0$,

$$
\lambda_{n k}\left(e^{-u}\right) \leq \frac{1}{2 \pi \lambda_{k}} \int_{-\infty}^{\infty} \frac{d v}{1+v^{2} \sigma_{n k}^{2} / 2}<\frac{\sqrt{2}}{\lambda_{k} \sigma_{n k}},
$$

and this completes the proof of Lemma 1.

The case $s=0,0=\lambda_{0}<\lambda_{1}<\lambda_{2}<\cdots$ of the following lemma is due to Hausdorff [4]. 
LEMMA 2. Let $\left\{\lambda_{n}\right\}$ be non-decreasing, and let $s$ be a non-negative integer. Then

$$
\lim _{n \rightarrow \infty} \sum_{k=s}^{n} \lambda_{n k}= \begin{cases}\alpha(1)-\alpha(0+) & \text { if } \lambda_{s}>0 \\ \alpha(1)-\alpha(0) & \text { if } \lambda_{s}=0\end{cases}
$$

and

$$
\lim _{n \rightarrow \infty} \lambda_{n s}= \begin{cases}0 & \text { if } \lambda_{s}>0 \\ \alpha(0+)-\alpha(0) & \text { if } \lambda_{s}=0\end{cases}
$$

Proof. It is known [3, Theorem 1(iv) and Theorem 2] that (15) holds with $s=0$ when $\alpha(t)$ is non-decreasing, and the general case of (15) with $s=0$ follows by expressing $\alpha(t)$ as the difference of two non-decreasing functions.

Next, suppose $s \geq 1$ and let $\tilde{\lambda}_{k}=\lambda_{k+s}$ for $k=0,1, \cdots$. Then, for $s \leq k \leq n$,

$$
\lambda_{n k}=\tilde{\lambda}_{n-s, k-s},
$$

$\tilde{\lambda}_{n k}$ being defined by (4) and (5) with $\left\{\lambda_{k}\right\}$ replaced by $\left\{\tilde{\lambda}_{k}\right\}$. Hence, as $n \rightarrow \infty$,

$$
\sum_{k=s}^{n} \lambda_{n k}=\sum_{k=0}^{n-s} \tilde{\lambda}_{n-s, k} \rightarrow \alpha(1)-\alpha(0+)
$$

by (15) with $s=0$, since $\tilde{\lambda}_{0}=\lambda_{s}>0$. This establishes (15) with $s \geq 0$.

To complete the proof of Lemma 2 we can deduce (16) from (15) by observing that, for $n>s \geq 0$,

$$
\lambda_{n s}=\sum_{k=s}^{n} \lambda_{n k}-\sum_{k=s+1}^{n} \lambda_{n k} .
$$

LEMma 3. Let $0 \leq \lambda_{0}<\lambda_{1}<\lambda_{2}<\cdots, 0<\delta<1 / 2$, and let $s$ be $a$ positive integer. Then there is an integer $N$ and a positive constant $M$ such that, for $n \geq N$,

$$
\sum_{k=s}^{n}\left(\int_{\delta}^{1-\delta} \lambda_{n k}(t)|d \alpha(t)|\right)^{2} \leq M \max \left(M_{1}(n, s), M_{2}(n, s)\right)
$$

where

$$
M_{1}(n, s)=\max _{s \leq k \leq n} \frac{e^{-\lambda_{s} \rho_{n k}}}{\lambda_{k}}
$$


and

$$
M_{2}(n, s)=\max _{\substack{s \leq k \leq n \\ \delta / 2 \leq e^{-\rho_{n k} \leq 1-\delta / 2}}} \frac{1}{\lambda_{k} \sigma_{n k}}
$$

Proof. Case 1. Suppose that $\lambda_{0}=0, s=1$. Let

$$
\omega_{n k}=\left(\left(1-\frac{\lambda_{1}}{\lambda_{k+1}}\right) \cdots\left(1-\frac{\lambda_{1}}{\lambda_{n}}\right)\right)^{1 / \lambda_{1}} \quad \text { for } 0 \leq k<n, \omega_{n n}=1
$$

Then, in view of (6), we have

$$
\begin{aligned}
\sum_{k=1}^{n}\left(\int_{\delta}^{1-\delta} \lambda_{n k}(t)|d \alpha(t)|\right)^{2} & \leq \int_{\delta}^{1-\delta}|d \alpha(t)| \cdot \max _{1 \leq k \leq n} \int_{\delta}^{1-\delta} \lambda_{n k}(t)|d \alpha(t)| \\
& \leq V_{\delta} \max \left(I_{1}, I_{2}\right)
\end{aligned}
$$

where $V_{\delta}=\int_{\delta}^{1-\delta}|d \alpha(t)|$

$$
I_{1}=\max _{\substack{1 \leq k \leq n \\\left|\omega_{n k}-1 / 2\right| \geq 1 / 2-3 \delta / 4}} \int_{\delta}^{1-\delta} \lambda_{n k}(t)|d \alpha(t)|
$$

and

$$
I_{2}=\max _{\substack{1 \leq k \leq n \\\left|\omega_{n k}-1 / 2\right|<1 / 2-3 \delta / 4}} \int_{\delta}^{1-\delta} \lambda_{n k}(t)|d \alpha(t)|
$$

To deal with $I_{1}$, let $f(t)$ be a twice continuously differentiable function on $[0,1]$ satisfying $0 \leq f(t) \leq 1, f(t)=1$ for $\left|t-\frac{1}{2}\right| \geq \frac{1}{2}-\frac{3 \delta}{4}, f(t)$ $=0$ for $\delta \leq t \leq 1-\delta$, and let

$$
B_{n}(f, t)=\sum_{k=0}^{n} \lambda_{n k}(t) f\left(\omega_{n k}\right)
$$

Then, by a result proved by Leviatan [8, Theorem 7],

$$
I_{1} \leq V_{\delta} \max _{\delta \leq t \leq 1-\delta}\left|B_{n}(f, t)-f(t)\right| \leq V_{\delta} K M_{1}(n, 1)
$$

where $K$ is a constant.

To deal with $I_{2}$ we note that, by Lemma 1,

$$
I_{2} \leq \max _{\substack{1 \leq k \leq n \\\left|\omega_{n k}-1 / 2\right|<1 / 2-3 \delta / 4}} \frac{V_{\delta} \sqrt{2}}{\lambda_{k} \sigma_{n k}}=\frac{V_{\delta} \sqrt{2}}{\lambda_{k(n)} \sigma_{n, k(n)}}
$$

where $k(n)$ is an integer satisfying $1 \leq k(n) \leq n, 3 \delta / 4<\omega_{n, k(n)}<1-$ $3 \delta / 4$. Since $\sum_{j=1}^{\infty} 1 / \lambda_{j}=\infty$, it follows that, for every fixed integer $j$, $\lim _{n \rightarrow \infty} \omega_{n j}=0$ and hence that $\lim _{n \rightarrow \infty} k(n)=\infty$. Further, since

$$
\log (1-x)=x+O\left(x^{2}\right) \text { for }|x| \leq 1 / 2 \text {, }
$$


we have that, for $k=k(n)$,

$$
\begin{aligned}
\omega_{n k} \sim \omega_{n, k-1} & =e^{-\rho_{n k}+O\left(\sigma_{n k}^{2}\right)} \\
& =e^{-\rho_{n k}+O\left(\rho_{n k} / \lambda_{k}\right)}=e^{-\rho_{n k}(1+o(1))} .
\end{aligned}
$$

Hence, for $n$ sufficiently large,

$$
\delta / 2<e^{-\rho_{n, k(n)}}<1-\delta / 2
$$

and thus

$$
I_{2} \leq V_{\delta} \sqrt{2} M_{2}(n, 1)
$$

This completes the proof of Case 1.

Case 2. Suppose that $\lambda_{0} \geq 0, s \geq 1$. Let

$$
\tilde{\lambda}_{0}=0, \quad \tilde{\lambda}_{k}=\lambda_{k+s-1} \quad \text { for } k=1,2, \ldots,
$$

and define $\tilde{\lambda}_{n, k}(t), \tilde{M}_{1}(n, s), \tilde{M}_{2}(n, s)$ by means of (4), (9), (17) and (18) with $\left\{\lambda_{k}\right\}$ replaced by $\left\{\tilde{\lambda}_{k}\right\}$. Then, for $n \geq k \geq s, 0 \leq t \leq 1$, we have

$$
\tilde{\lambda}_{n-s+1, k-s+1}(t)=\lambda_{n k}(t),
$$

and hence, by Case 1,

$$
\begin{gathered}
\sum_{k=s}^{n}\left(\int_{\delta}^{1-\delta} \lambda_{n k}(t)|d \alpha(t)|\right)^{2}=\sum_{r=1}^{n-s+1}\left(\int_{\delta}^{1-\delta} \tilde{\lambda}_{n-s+1, r}(t)|d \alpha(t)|\right)^{2} \\
\leq M \max \left(\tilde{M}_{1}(n-s+1,1), \tilde{M}_{2}(n-s+1,1)\right) \\
=M \max \left(M_{1}(n, s), M_{2}(n, s)\right) .
\end{gathered}
$$

This completes the proof of Lemma 3.

LEMmA 4. Let $0 \leq \lambda_{0}<\lambda_{1}<\lambda_{2}<\cdots, 0<\delta<1 / 2, s \geq 2, \lambda_{s}>M$ +1 , and let $\lambda$ satisfy either (10) or (11) with the same $M$ for $k \geq s-1$. Then

$$
\lim _{n \rightarrow \infty} \log n \sum_{k=s}^{n}\left(\int_{\delta}^{1-\delta} \lambda_{n k}(t)|d \alpha(t)|\right)^{2}=0
$$

Proof. Case 1. Suppose that $\lambda$ satisfies (10) for $k \geq s-1$, and that $n \geq k \geq s$. Then $\lambda_{n} \geq \lambda_{s}+m(n-s)$, and

$$
M \rho_{n k} \geq \sum_{j=k}^{n} \frac{\lambda_{j+1}-\lambda_{j}}{\lambda_{J} \log \lambda_{J}} \geq \sum_{j=k}^{n} \int_{\lambda_{J}}^{\lambda_{j}+1} \frac{d x}{x \log x}=\log \frac{\log \lambda_{n+1}}{\log \lambda_{k}}
$$


Hence

$$
\frac{e^{-\lambda_{s} \rho_{n k}}}{\lambda_{k}} \leq \frac{1}{\lambda_{k}}\left(\frac{\log \lambda_{k}}{\log \lambda_{n+1}}\right)^{\lambda_{s} / M}
$$

and so

$$
M_{1}(n, s)=O\left(\left(\log \lambda_{n+1}\right)^{-\lambda_{s} / M}\right)=o\left(\frac{1}{\log n}\right) .
$$

Suppose now that

$$
\frac{\delta}{2} \leq e^{-\rho_{n k}} \leq 1-\frac{\delta}{2}
$$

Then

$$
m \log \frac{2}{2-\delta} \leq m \rho_{n k} \leq \sum_{J=k}^{n} \frac{\lambda_{J}-\lambda_{j-1}}{\lambda_{j}} \leq \sum_{J=k}^{n} \int_{\lambda_{J-1}}^{\lambda_{J}} \frac{d x}{x}=\log \frac{\lambda_{n}}{\lambda_{k-1}}
$$

so that $\lambda_{k-1} \leq(1-\delta / 2)^{m} \lambda_{n}$ and hence, by (10), we have that

$$
\lambda_{k} \leq \lambda_{k-1}+M \log \lambda_{k} \leq\left(1-\frac{\delta}{2}\right)^{m} \lambda_{n}+M \log \lambda_{n} .
$$

Further, by (19) and (21),

$$
M \log \frac{2}{\delta} \geq \log \frac{\log \lambda_{n+1}}{\log \lambda_{k}}
$$

and so

$$
\lambda_{k} \geq \lambda_{n+1}^{\varepsilon}
$$

where $\varepsilon=(\delta / 2)^{M}$.

Next, let $f(x)=1 / x \log x$ so that

$$
f^{\prime}(x)=\frac{1}{x^{2} \log x}\left(1+\frac{1}{\log x}\right) \leq \frac{c}{x^{2} \log x}
$$

for $x \geq \lambda_{s}$ where $c=1+1 / \log \lambda_{s}>0$. Hence, by (10), (22) and (23),

$$
\begin{aligned}
c M\left(\lambda_{k} \sigma_{n k}\right)^{2} & \geq c \lambda_{k}^{2} \sum_{j=k}^{n} \frac{\lambda_{j+1}-\lambda_{j}}{\lambda_{j}^{2} \log \lambda_{j}} \\
& \geq \lambda_{k}^{2} \sum_{j=k}^{n} \int_{\lambda_{j}}^{\lambda_{j+1}} \frac{c d x}{x^{2} \log x} \geq \lambda_{k}^{2} \int_{\lambda_{k}}^{\lambda_{n+1}} f^{\prime}(x) d x \\
& =\frac{\lambda_{k}}{\log \lambda_{k}}\left(1-\frac{\lambda_{k} \log \lambda_{k}}{\lambda_{n+1} \log \lambda_{n+1}}\right) \\
& \geq \frac{\lambda_{n}^{\varepsilon}}{\log \lambda_{n}}\left(1-(1-\delta / 2)^{m}-\frac{M \log \lambda_{n}}{\lambda_{n}}\right) .
\end{aligned}
$$


Consequently

$$
\begin{aligned}
M_{2}(n, s) & =O\left(\lambda_{n}^{-\varepsilon / 2} \log ^{1 / 2} \lambda_{n}\right)=O\left(\lambda_{n}^{-\varepsilon / 4}\right)=O\left(n^{-\varepsilon / 4}\right) \\
& =o\left(\frac{1}{\log n}\right) .
\end{aligned}
$$

The desired conclusion in Case 1 now follows from (20) and (24), by Lemma 3.

Case 2. Suppose that $\lambda$ satisfies (11) for $k \geq s-1$ and that $n \geq k \geq s$. Then

$$
M \rho_{n k} \geq \sum_{j=k}^{n} \frac{\lambda_{j+1}-\lambda_{j}}{\lambda_{j}} \geq \sum_{j=k}^{n} \int_{\lambda_{j}}^{\lambda_{j+1}} \frac{d x}{x}=\log \frac{\lambda_{n+1}}{\lambda_{k}} .
$$

Hence, since $\lambda_{s}>M+1$,

$$
\frac{e^{-\lambda_{s} \rho_{n k}}}{\lambda_{k}} \leq \frac{1}{\lambda_{k}}\left(\frac{\lambda_{k}}{\lambda_{n+1}}\right)^{\lambda_{s} / M} \leq \frac{1}{\lambda_{n}}
$$

and so

$$
M_{1}(n, s) \leq \frac{1}{\lambda_{n}}=o\left(\frac{1}{\log n}\right) .
$$

Suppose now that (21) holds. Then, by (25),

$$
\lambda_{k} \geq \lambda_{n+1}(\delta / 2)^{M},
$$

and hence

$$
\begin{aligned}
\lambda_{k} \sigma_{n k} & \geq \lambda_{k}\left(\frac{\rho_{n k}}{\lambda_{n}}\right)^{1 / 2} \geq \lambda_{k}\left(\frac{1}{\lambda_{n}} \log \frac{2}{2-\delta}\right)^{1 / 2} \\
& \geq\left(\frac{\delta}{2}\right)^{M}\left(\log \frac{2}{2-\delta}\right)^{1 / 2} \lambda_{n}^{1 / 2} .
\end{aligned}
$$

Consequently

$$
M_{2}(n, s)=O\left(\lambda_{n}^{-1 / 2}\right)=o\left(\frac{1}{\log n}\right) .
$$

The desired conclusion now follows from (26) and (27), by Lemma 3, and this completes the proof of Lemma 4.

3. Proof of Theorem 1. Suppose that $n \geq k \geq s$ and that $r=$ $3,4, \ldots$ Let

$$
\lambda_{n k}^{r}=\int_{1 / r}^{1-1 / r} \lambda_{n k}(t) d \alpha(t)
$$


Let $\left\{X_{n}\right\}$ be a sequence of random variables satisfying (1) and (2) with $p=2$, and let

$$
T_{n}=\sum_{k=s}^{n} \lambda_{n k} X_{k}, \quad T_{n}^{r}=\sum_{k=s}^{n} \lambda_{n k}^{r} X_{k} .
$$

By Lemma 4, we have, subject to either (10) or (11), that

$$
\log n \sum_{k=s}^{n}\left(\lambda_{n k}^{r}\right)^{2} \rightarrow 0 \quad \text { as } n \rightarrow \infty .
$$

Hence, by Theorem A,

$$
T_{n}^{r} \rightarrow 0 \text { a.s. as } n \rightarrow \infty \text {. }
$$

Let $\Omega_{r}$ be the subset of $\Omega$ on which $T_{n}^{r} \rightarrow 0$ and $\left|X_{r}\right| \leq M$, and let $\Omega_{0}=\bigcap_{r=3}^{\infty} \Omega_{r}$. Then

$$
\begin{aligned}
T_{n}-T_{n}^{r} & =\sum_{k=s}^{n} X_{k}\left\{\int_{0}^{1} \lambda_{n k}(t) d \alpha(t)-\int_{1 / r}^{1-1 / r} \lambda_{n k}(t) d \alpha(t)\right\} \\
& =\sum_{k=s}^{n} X_{k}\left(\int_{0}^{1 / r}+\int_{1-1 / r}^{1}\right) \lambda_{n k}(t) d \alpha(t),
\end{aligned}
$$

and hence, in view of (6), on $\Omega_{0}$

$$
\left|T_{n}-T_{n}^{r}\right| \leq M\left(\int_{0}^{1 / r}+\int_{1-1 / r}^{1}\right)|d \alpha(t)| \rightarrow 0 \quad \text { as } r \rightarrow \infty,
$$

since $\alpha(0+)=\alpha(0)$ and $\alpha(1-)=\alpha(1)$. Thus

$$
\lim _{r \rightarrow \infty} T_{n}^{r}=T_{n} \quad \text { on } \Omega_{0} \text { uniformly in } n \text { for } n \geq s .
$$

On the other hand

$$
\lim _{n \rightarrow \infty} T_{n}^{r}=0 \quad \text { on } \Omega_{0} \text { for } r \geq 3
$$

It follows that

$$
\lim _{n \rightarrow \infty} T_{n}=\lim _{n \rightarrow \infty} \lim _{r \rightarrow \infty} T_{n}^{r}=\lim _{r \rightarrow \infty} \lim _{n \rightarrow \infty} T_{n}^{r}=0 \quad \text { on } \Omega_{0} .
$$

i.e., $T_{n} \rightarrow 0$ a.s.

Since $\alpha(0)=\alpha(0+)$ we have, by Lemma 2 , that $\lim _{n \rightarrow \infty} \lambda_{n k}=0$ for $k \geq 0$. Consequently

$$
\sum_{k=0}^{n} \lambda_{n k} X_{k} \rightarrow 0 \quad \text { a.s. }
$$

and so $H(\lambda, \alpha) \in \Gamma_{2}$. 
Finally, the additional condition $\alpha(1)-\alpha(0)=1$ ensures, by Lemma 2 , that

$$
\lim _{n \rightarrow \infty} \sum_{k=0}^{n} \lambda_{n k}=1
$$

and hence that $H(\lambda, \alpha) \in(B P)$.

4. Proof of Theorem 2. Let $0 \leq k \leq n$. By (5), we have that

$$
\lambda_{n k}=\int_{0}^{1} \lambda_{n k}(t) \beta(t) d t .
$$

First, suppose that (12) holds. Then, by Hölder's inequality and (7),

$$
\begin{aligned}
\left|\lambda_{n k}\right|^{p} & \leq\left(\int_{0}^{1} \lambda_{n k}(t)|\beta(t)|^{p} d t\right)\left(\int_{0}^{1} \lambda_{n k}(t) d t\right)^{p-1} \\
& =\left(\frac{d_{k}}{D_{n}}\right)^{p-1} \int_{0}^{1} \lambda_{n k}(t)|\beta(t)|^{p} d t .
\end{aligned}
$$

Hence, by (6) and (12),

$$
\begin{aligned}
\left(\sum_{k=0}^{n}\left|\lambda_{n k}\right|^{p}\right)^{1 /(p-1)} & \leq \frac{1}{D_{n}}\left(\int_{0}^{1}|\beta(t)|^{p} d t \sum_{k=0}^{n} d_{k}^{p-1} \lambda_{n k}(t)\right)^{1 /(p-1)} \\
& \leq \max _{0 \leq k \leq n} d_{k} \cdot \frac{\|\beta\|_{p}^{p /(p-1)}}{D_{n}}=o\left(\frac{1}{\log n}\right) .
\end{aligned}
$$

It follows, by Theorem A, that $H(\lambda, \alpha) \in \Gamma_{p}$.

Next, suppose that (13) holds. Then, by (7),

$$
\left|\lambda_{n k}\right| \leq\|\beta\|_{\infty} \int_{0}^{1} \lambda_{n k}(t) d t=\|\beta\|_{\infty} \frac{d_{k}}{D_{n}},
$$

and hence

$$
\left(\sum_{k=0}^{n}\left|\lambda_{n k}\right|^{p}\right)^{1 /(p-1)} \leq\|\beta\|_{\infty}^{p /(p-1)}\left(\sum_{k=0}^{n}\left(\frac{d_{k}}{D_{n}}\right)^{p}\right)^{1 /(p-1)}=o\left(\frac{1}{\log n}\right) .
$$

Thus, by Theorem A, we have that $H(\lambda, \alpha) \in \Gamma_{p}$.

In view of Lemma 2, the additional conditions $\left\{\lambda_{n}\right\}$ monotonic and $\alpha(1)=1$, ensure that

$$
\lim _{n \rightarrow \infty} \sum_{k=0}^{n} \lambda_{n k}=1
$$

and hence that $H(\lambda, \alpha) \in(B P)$.

This completes the proof of Theorem 2 . 


\section{REFERENCES}

1. D. Borwein, Matrix transformations of weakly multiplicative sequences of random variables, J. London Math. Soc., (2) 23 (1981), 363-371.

2. D. Borwein and F. P. A. Cass, Weighted means, generalised Hausdorff matrices and the Borel property, Acta Sci. Math., 37 (1981), 29-34.

3. D. Borwein and A. Jakimovski, Generalization of the Hausdorff moment problem, Canad. J. Math., 33 (1981), 946-960.

4. F. Hausdorff, Summationsmethoden und Momentfolgen II, Math. Z., 9 (1921), 280-299.

5. J. D. Hill, The Borel property of summability methods, Pacific J. Math., 1 (1951), 399-409.

6. __ Remarks on the Borel property, Pacific J. Math., 4 (1954), 227-242.

7. A. Jakimovski, Generalized Bernstein polynomials for discontinuous and convex functions, J. D'Analyse Mathématique, 23 (1970), 171-183.

8. D. Leviatan, On the remainder in the approximation of functions by Bernstein-type operators, J. Approximation Theory, 2 (1969), 400-409.

9. M. Liu and B. E. Rhoades, Some properties of generalised Hausdorff matrices, Houston J. Math., 2 (1976), 239-256.

Received September 8, 1981 and in revised form March 11, 1982. Supported in part by the Natural Sciences and Engineering Research Council of Canada, Grant A-2983.

THE UNIVERSITY OF WeSTERN ONTARIO

LONDON, ONTARIO N6A 5B7

AND

TeL-Aviv UnIVERSITY

Ramat-Aviv, Tel-Aviv, ISRAEL 


\section{PACIFIC JOURNAL OF MATHEMATICS}

\section{EDITORS}

Donald BabbitT (Managing Editor)

University of California

Los Angeles, CA 90024

Hugo Rossi

University of Utah

Salt Lake City, UT 84112

C. C. Moore and Arthur Ogus

University of California

Berkeley, CA 94720
J. DugunduI

Department of Mathematics

University of Southern California

Los Angeles, CA 90089-1113

R. FinN and H. SAmelson

Stanford University

Stanford, CA 94305

\section{ASSOCIATE EDITORS}
R. ARENS
E. F. BECKENBACH
B. H. NEUMANN
F. WOLF
K. YosHIDA (1906-1982)

\section{SUPPORTING INSTITUTIONS}

UNIVERSITY OF ARIZONA

UNIVERSITY OF BRITISH COLUMBIA

CALIFORNIA INSTITUTE OF TECHNOLOGY

UNIVERSITY OF CALIFORNIA

MONTANA STATE UNIVERSITY

UNIVERSITY OF NEVADA, RENO

NEW MEXICO STATE UNIVERSITY

OREGON STATE UNIVERSITY
UNIVERSITY OF OREGON

UNIVERSITY OF SOUTHERN CALIFORNIA

STANFORD UNIVERSITY

UNIVERSITY OF HAWAII

UNIVERSITY OF TOKYO

UNIVERSITY OF UTAH

WASHINGTON STATE UNIVERSITY

UNIVERSITY OF WASHINGTON 


\section{Pacific Journal of Mathematics}

Vol. 107, No. $1 \quad$ January, 1983

John Kelly Beem and Phillip E. Parker, Klein-Gordon solvability and the

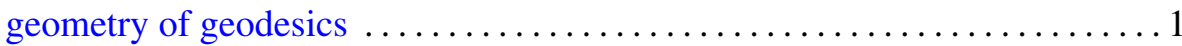

David Borwein and Amnon Jakimovski, Transformations of certain

sequences of random variables by generalized Hausdorff matrices ..... 15

Willy Brandal and Erol Barbut, Localizations of torsion theories . . . . . . . 227

John David Brillhart, Paul Erdős and Richard Patrick Morton, On sums

of Rudin-Shapiro coefficients. II ........................... 39

Martin Lloyd Brown, A note on tamely ramified extensions of rings $\ldots \ldots \ldots 71$

Chang P'ao Ch'ên, A generalization of the Gleason-Kahane-Żelazko

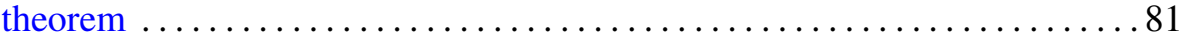

I. P. de Guzman, Annihilator alternative algebras $\ldots \ldots \ldots \ldots \ldots \ldots$. . . . 89

Ralph Jay De Laubenfels, Extensions of $d / d x$ that generate uniformly

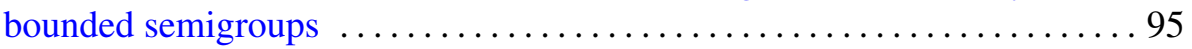

Patrick Ronald Halpin, Some Poincaré series related to identities of $2 \times 2$

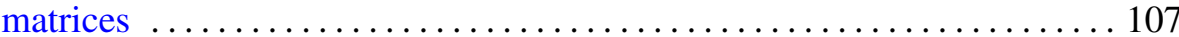

Fumio Hiai, Masanori Ohya and Makoto Tsukada, Sufficiency and

relative entropy in $*$-algebras with applications in quantum systems . . . 117

Dean Robert Hickerson, Splittings of finite groups $\ldots \ldots \ldots \ldots \ldots \ldots \ldots 14$

Jon Lee Johnson, Integral closure and generalized transforms in graded

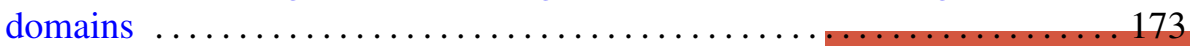

Maria Grazia Marinari, Francesco Odetti and Mario Raimondo, Affine

curves over an algebraically nonclosed field . ................. 179

Douglas Shelby Meadows, Explicit PL self-knottings and the structure of

PL homotopy complex projective spaces $\ldots \ldots \ldots \ldots \ldots \ldots \ldots \ldots \ldots$

Charles Kimbrough Megibben, III, Crawley's problem on the unique

$\omega$-elongation of $p$-groups is undecidable .................... 205

Mary Elizabeth Schaps, Versal determinantal deformations $\ldots \ldots \ldots \ldots 213$

Stephen Scheinberg, Gauthier's localization theorem on meromorphic

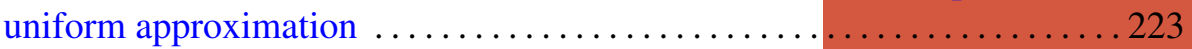

Peter Frederick Stiller, On the uniformization of certain curves . . . . . . . 229

Ernest Lester Stitzinger, Engel's theorem for a class of algebras . . . . . . . . 245

Emery Thomas, On the zeta function for function fields over $F_{p} \ldots \ldots \ldots 251$ 